\title{
Fast Drug Scheduling Optimization Approach for Cancer Chemotherapy
}

\author{
Yong Liang ${ }^{1}$, Kwong-Sak Leung ${ }^{2}$, and Tony Shu Kam Mok ${ }^{3}$ \\ ${ }^{1}$ Department of Computer Science, Shantou University, China \\ ${ }^{2}$ Department of Computer Science \& Engineering, \\ The Chinese University of Hong Kong, HK \\ ${ }^{3}$ Department of Clinical Oncology, \\ The Chinese University of Hong Kong, HK \\ yliang@stu.edu.cn, ksleung@cse.cuhk.edu.hk, tony@clo.cuhk.edu.hk
}

\begin{abstract}
In this paper, we propose a novel fast evolutionary algorithm - cycle-wise genetic algorithm (CWGA) based on the theoretical analyses of a drug scheduling mathematical model for cancer chemotherapy. CWGA is more efficient than other existing algorithms to solve the drug scheduling optimization problem. Moreover, its simulation results match well with the clinical treatment experience, and can provide much more drug scheduling policies for a doctor to choose depending on the particular conditions of the patients. CWGA also can be widely used to solve other kinds of the real dynamic systems.
\end{abstract}

Keywords: Genetic algorithm, Drug scheduling model.

\section{Introduction}

An important target for cancer chemotherapy is to maximally kill tumor cells for a fixed treatment period. So drug scheduling is essential in cancer chemotherapy. Martin [7] have proposed the optimal drug scheduling model by the following differential equations:

$$
\begin{aligned}
\frac{d x_{1}}{d t} & =-\lambda x_{1}+k\left(x_{2}-\beta\right) H\left(x_{2}-\beta\right) \\
\frac{d x_{2}}{d t} & =u-\gamma x_{2} \\
\frac{d x_{3}}{d t} & =x_{2}
\end{aligned}
$$

with the initial state $x^{T}(0)=[\ln (100), 0,0]$, the parameters $\lambda=9.9 \times 10^{-4}$, $k=8.4 \times 10^{-3}, \beta=10, \gamma=0.27$ and:

$$
H\left(x_{2}-\beta\right)=\left\{\begin{array}{lll}
1, & \text { if } & x_{2} \geq \beta \\
0, & \text { if } & x_{2} \leq \beta
\end{array}\right.
$$

where $x_{1}$ is a transformed variable that is inversely related to the mass of the tumor. The tumor mass is given by $N=10^{12} \times \exp \left(-x_{1}\right)$ cells, and the initial 
tumor cell population is set at $10^{10}$ cells [7]. The variable $x_{2}$ is the drug concentration in the body in drug units $(D)$ and $x_{3}$ is the cumulative drug toxicity in the body. Equation (1) describes the net change in the tumor cell population per unit time. The first term on the right-hand side of Equation (1) describes the increase in cells due to cell proliferation and the second term describes the decrease in cells due to the drug. The parameter $\lambda$ is a positive constant related to the growth speed of the cancer cells, and $k$ is the proportion of tumor cells killed per unit time per unit drug concentration which is assumed to be a positive constant. The implication of the function described in Equation (4) is that there is a threshold drug concentration level, $\beta$ below which the number of the killed tumor cells is smaller than the number of the reproduced tumor cells, and the drug is not efficient. Equation (2) describes the net increase in the drug concentration at the cancer site. The variable $u$ is the rate of delivery of the drug, and the half-life of the drug is $\ln (2) / \gamma$, where $\gamma$ is the biochemical character parameter of the drug. It is assumed that the drug is delivered by infusion, and there is an instantaneous mixing of the drug with plasma, as well as an immediate delivery of the drug to the cancer site. These assumptions represent approximations based on the relative amount of time. It takes for the aforementioned activities to occur with respect to the total amount of time over which the treatment is administered. Equation (3) relates the cumulative drug toxicity to the drug concentration, e.g., the cumulative effect is the integral of the drug concentration over the period of exposure.

The performance index [7] to be maximized is:

$$
I=x_{1}\left(t_{f}\right)
$$

where the final time $t_{f}=84$ days. The control optimization is performed subject to constraints on the drug delivery: $u \geq 0$, and on the state variables: $x_{2} \leq 50$, $x_{3} \leq 2.1 \times 10^{3}$.

Cancer chemotherapy is a systemic treatment, so the action of the chemotherapeutic agent is not restricted to the tumor site. Any of the body organs are liable to injury. This is on contrast to the localized treatments, such as surgery or radiotherapy. Therefore, the constraints on the drug concentration $x_{2}$ and the cumulative drug toxicity $x_{3}$ are to ensure that the patient can tolerate the toxic side effects of the drug. Drug resistance is considered to be a significant factor in chemotherapeutic failure [8] and it has been shown that the drug resistant cells are likely to increase as the tumor burden increases [6]. In order to reduce the likelihood of the emergence of drug resistant cells, the tumor size is forced to reduce by at least $50 \%$ every 3 weeks, so that: $x_{1}(21) \geq \ln (200), x_{1}(42) \geq \ln (400)$, $x_{1}(63) \geq \ln (800)$.

Many researchers have applied different optimization methods to improve the results of the drug scheduling model [1] [3] [4] 6] [7] 8] [10] [11. Through analyzing the experimental results from the existing model, there are two obvious unreasonable outcomes in the optimal drug scheduling policies: $(i)$ unreasonable timing for the first treatment; and $(i i)$ three point constraints cannot improve the efficiency of the cancer treatment. To solve the unreasonable problems and 
accurately describe the time course of the cumulative drug toxicity $x_{3}$ in the body, in [5], we have modified the third equation in Martin's model as follows:

$$
\frac{d x_{3}}{d t}=x_{2}-\eta_{L} \times x_{3}\left(1-\frac{x_{3}}{2 \theta}\right)-\eta_{G} \times x_{3} \ln \left(\frac{2 \theta}{x_{3}}\right)-\eta_{E} \times x_{3},
$$

where $\eta_{L}, \eta_{G}, \eta_{E}$ are nonnegative constants. We set the constraint of the cumulative drug toxicity to $x_{3}<\theta$.

Equation (6) describes the net change of the cumulative drug toxicity $x_{3}$ per unit time. In the right-hand side of this equation, the first term $x_{2}$ describes the increase of the cumulative drug toxicity $x_{3}$ due to the drug concentration $x_{2}$. The second, third and fourth terms can be called logistic, Gompertz and exponential drug toxicity clearance functions respectively, which describe the decreases in the drug toxicity due to the clearance in the body. As you know, the liver and kidney are the primary detoxification and elimination organs - they eliminate the drug toxicity from our body. Drugs passing through the liver are eliminated in a chemically altered (metabolized) form in the bile. Whenever, drug metabolism or movement across the liver involves an active process, then the likelihood of saturable kinetics exists. Thus, the logistic and Gompertz functions give the fit for saturable toxic elimination processes in the liver. On the other hand, drugs, particularly water-soluble drugs and their metabolites, are also eliminated by the kidney in urine. The kidney is a filter that cleanses toxins from our blood and its ability to excrete the toxic compounds depends on the amount of the drug toxicity in the bloodstream. Therefore, the renal excretion (i.e., filtration and passive reabsorption) may be best considered to the nonsaturable mechanisms and described by the exponential function. We combine Equation (6) with Equations (1) and (2) to construct a renewed drug scheduling model. It matches well with the clinical treatment knowledge and experience.

The rest of this paper is organized as follows. Section 2 analyzes the optimal solutions obtained by the renewed drug scheduling model. Section 3 presents the theoretical analysis of the drug scheduling model. Section 4 describes a fast evolutionary algorithm — cycle-wise genetic algorithm (CWGA) to solve the drug scheduling problem. Section 5 provides the simulation results under the new optimization approach. The paper conclusion and future work are summarized in Section 6.

\section{Analysis of the Experimental Results of the Renewed Model}

In our previous work, we use our proposed adaptive elitist-population based genetic algorithm (AEGA), which is an efficient multimodal optimization algorithm [2, to implement the optimization of the drug scheduling model for exploring multiply efficient drug scheduling policies. In the drug scheduling problem as described in Section 1 , there are 84 control variables to be optimized, which represent the dosage levels for the 84 days. The drug scheduling model is a high dimensional and multimodal optimization problem. Here we have proposed a cycle-wise variable representation to accurately and efficiently describe the drug scheduling policy. 
The cycle-wise variable representation includes two parts: a front and a cyclic parts. For example, the cycle-wise variable representation is $\{57.05,13.5,0,10.8$, $80 \times \overline{21.5}\}$ that means giving the drug doses 57.05, 13.5, 0, 10.8 at the first four days respectively, and then repeated 80 times giving the drug dose 21.5 every two days. Its front part is $\{57.05,13.5,0,10.8\}$. Its cyclic part is $80 \times \overline{21.5}$, which consists of the number of cycles 80 and the repetend $\overline{21.5}$. The cycle-wise variable representation is very suitable for the drug scheduling problem. Because in the first few days of the treatment period, the patient's body may not have adapted to the drug, but it is important to kill as much tumor cells as possible, the drug doses will be adjusted day by day. We use the front part to represent the drug doses in this initial period. Then when the patient's body gradually gets used to the drug, the drug administration schedule will follow a fixed cycle and a fixed dose pattern, which is suitably represented by the cycle-part.

Fig.1-(a) and (b) show the multiple optimal solutions, which are explored by AEGA from our renewed drug scheduling model. We also used other optimization algorithm — iterative dynamic programming (IDP) without the cycle-wise variable representation [6] to solve the renewed model. The global optimal solution obtained by IDP is as same as Fig.1-(a) shown.
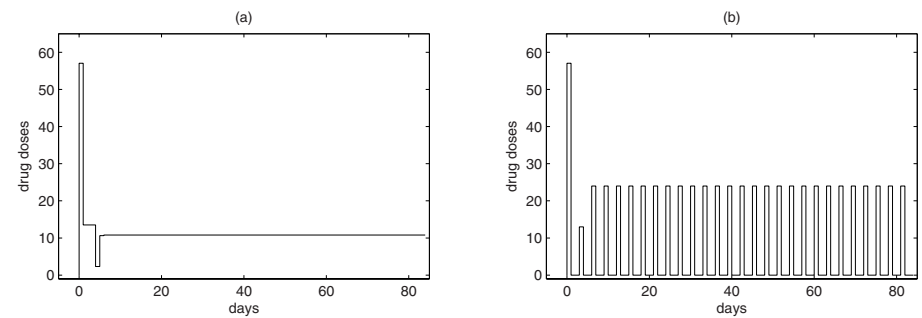

Fig. 1. The multiple optimal solutions obtained by the renewed drug scheduling model $\left(\eta_{L}=0, \eta_{G}=0, \eta_{E}=0.4\right)$. (a): global optimal solution; (b): second optimal solution.

We believe that the cycle-wise variable representation is suitable and consistent with the renewed drug scheduling model. So we do the theoretical analysis of this model in the next section. The results show that the cycle-wise variable representation can be used to predict the treatment results of the drug and significantly reduce the computational complexity of the optimization algorithm. Moreover, it is more consistent with the clinical experience.

\section{Theoretical Analysis of the Renewed Drug Scheduling Model}

In the renewed drug scheduling model, Equation (6) is a higher order differential equation. We can write it as a system with a very simple change of variable. We define the following three new functions.

$$
y_{1}=\eta_{L} \times x_{3}\left(1-\frac{x_{3}}{2 \theta}\right)
$$




$$
\begin{aligned}
& y_{2}=\eta_{G} \times x_{3} \ln \left(\frac{2 \theta}{x_{3}}\right) \\
& y_{3}=\eta_{E} \times x_{3}
\end{aligned}
$$

Equation (6) can then be wrote the following system of differential equations.

$$
\frac{d y_{i}}{d t}=\tau_{i} \times x_{2}-y_{i}, \quad i=1,2,3
$$

We combine Equations (1) and (2) with the differential equations (10) instead of Equation (6). The renewed drug scheduling model can then be considered as the first order, linear and homogeneous systems of differential equations.

Theorem 1. In the drug scheduling model, suppose the giving drug doses $u$ is a cycle-wise variable with a cycle $\Delta t$ (giving drug at $t_{0}, t_{0}+\Delta t, t_{0}+2 \Delta t, \ldots$, days), then the maximal values of the drug concentration $x_{2}$ and the drug cumulative toxicity $y_{1}+y_{2}+y_{3}$ are approximately equal in each $\Delta t$ respectively (e.g., $x_{2}\left(t_{0}\right) \approx$ $\left.x_{2}\left(t_{0}+\Delta t\right) \approx x_{2}\left(t_{0}+2 \Delta t\right) \ldots\right)$.

Proof : Suppose giving drug at $t_{0}, t_{0}+\Delta t, t_{0}+2 \Delta t, \ldots$, days in the cycle part, then the drug concentration $x_{2}$ or the drug cumulative toxicity $y_{1}+y_{2}+y_{3}$ will meet its constraint at these days. If supposing that $x_{2}$ meets its constraint, this means that $x_{2}$ monotonously increases and reaches its maximal value at times $t_{0}, t_{0}+\Delta t, t_{0}+2 \Delta t, \ldots$, then,

$$
\begin{aligned}
& \frac{d x_{2}}{d t}\left(t_{0}\right) \approx \frac{d x_{2}}{d t}\left(t_{0}+\Delta t\right) \approx \frac{d x_{2}}{d t}\left(t_{0}+2 \Delta t\right) \approx 0 \\
& \begin{aligned}
u\left(t_{0}\right)-\lambda x_{2}\left(t_{0}\right) & \approx u\left(t_{0}+\Delta t\right)-\lambda x_{2}\left(t_{0}+\Delta t\right) \\
& \approx u\left(t_{0}+2 \Delta t\right)-\lambda x_{2}\left(t_{0}+2 \Delta t\right)
\end{aligned}
\end{aligned}
$$

Since $u\left(t_{0}\right)=u\left(t_{0}+\Delta t\right)=u\left(t_{0}+2 \Delta t\right)$, then

$$
x_{2}\left(t_{0}\right) \approx x_{2}\left(t_{0}+\Delta t\right) \approx x_{2}\left(t_{0}+2 \Delta t\right)
$$

In the same way, we can get the same results of the drug toxicity $y_{1}+y_{2}+y_{3}$ as the drug concentration $x_{2}$.

In the drug scheduling model, there is not the constraint for the number of cancer cells $x_{1}$. In equation (1), $x_{2}$ must be larger than $\beta$ for any efficient drug scheduling policy, then $k\left(x_{2}-\beta\right) \gg \lambda x_{1}$. We can evaluate the number of cancer cells at the end of the treatment period using the formula $x_{1}\left(t_{0}+k \times \Delta t\right)=$ $x_{1}\left(t_{0}\right)+k \times\left(x_{1}\left(t_{0}+\Delta t\right)-x_{1}\left(t_{0}\right)\right)$. Based on the theoretical analysis of the drug scheduling model, we can only calculate the values of $x_{1}, x_{2}$ and $y_{1}+y_{2}+y_{3}$ in front part and first two cycles of the cycle part, then using them evaluate the values of $x_{1}, x_{2}$ and $y_{1}+y_{2}+y_{3}$ at the end of the treatment period. Thus, the computational complexity of the algorithm will be significantly reduced to solve the drug scheduling model. 


\section{Optimization of Drug Scheduling Model Via CWGA}

In this section we propose a new genetic algorithm - a cycle-wise genetic algorithm (CWGA) to implement the optimization of the drug scheduling model for exploring multiply efficient drug scheduling policies.

In order to successfully explore multiple optimal solutions of the drug scheduling model, several rules for applying the CWGA based on our proposed adaptive elitist- population search technique [2] are made as follows:

- Use the cycle-wise representation to keep the scheduling freedom and improve the efficiencies of algorithm.

- Use the front part and the first two cycles of the cycle-wise representation to evaluate the fitness of the drug scheduling policy to significantly reduce the computational complexity of the algorithm.

- Use the adaptive elitist-population search technique in the crossover operator to reduce the redundancy of the population.

- Use the adaptive elitist-population search technique in the mutation operator to increase the diversity of the population.

- Adaptively adjust the population size to optimally use the elitist individuals to explore multiple optima.

Following these rules, the CWGA for the drug scheduling model is implemented as follows:

1. Set $t=0$ and initialize a chromosome population $P(t)$

(uniform random initialization within the bounds);

2. Evaluate $P(t)$ using the fitness measure;

3. While (termination condition not satisfied) Do

(a) Elitist crossover operation to generate $P(t+1)$;

i. check the dissimilarity of the randomly selected parents $p_{i}$ and $p_{j}$;

ii. if the parents $p_{i}$ and $p_{j}$ are similar, the elitist operation conserves the better one of them for the next generation; else, according to multi-point crossover operation, generates 6 offspring, and selects the better two from the parents and their offspring to the next generation;

(b) Elitist mutation operation to generate $P(t+1)$;

i. according to the one-point mutation operation, generate the offspring $c_{i}$ from the parent $p_{i}$;

ii. if $p_{i}$ and $c_{i}$ are dissimilar, the elitist operation conserves $p_{i}$ and $c_{i}$ together for the next generation; else, selects the better one of them to the next generation;

4. Evaluate $P(t+1)$;

5. Stop if the termination condition is satisfied; otherwise, go to Step 3. 


\section{Experimental Results and Comparisons}

The drug scheduling problem were simulated using the CWGA with the following parameters: initial population size $=100$; maximal number of generations $=10000$; crossover rate $=1.0 ;$ mutation rate $=1.0$ and the distance threshold $\sigma_{s}=10$. We set the parameters $\eta_{L}=0.01, \eta_{G}=0.01, \eta_{E}=0.38$ in the model. The drug scheduling model was simulated using numerical differentiation method of Runge-Kutta [9], with a small time interval of 0.1 day for good accuracy.

Optimizing the renewed drug scheduling model via CWGA for 50 times can consistently obtain 6 most efficient drug scheduling policies. These results are listed in Table 1. For example, Fig. 2 and 3 show the control variable $u$, the number of cancer cells (inversely related to the best performance index $x_{1}$ ), the change processes of the drug concentration $x_{2}$ and the cumulative drug toxicity $x_{3}$ of the first and sixth optimal policies. The 6 best results all satisfy the three point constraints, and therefore it is not necessary to find the special solutions for the new model with the three point constraints separately.

Table 1. The most efficient drug scheduling policies obtained by the renewed model

\begin{tabular}{lc}
\hline The most efficient drug scheduling policies & Cancer cells \\
\hline (1): $\{92.21,(83 \times \overline{10.8})\}$ & 21 \\
(2): $\{132.69,0,22.57,0,8.63,0,24.52,(38 \times \overline{0,21.02}), 8.22\}$ & 32 \\
(3): $\{132.69,(2 \times \overline{0}), 31.5,(2 \times \overline{0}), 24.5,(25 \times \overline{(2 \times \overline{0}), 29.89}), 0,25.24\}$ & 68 \\
(4): $\{132.69,(3 \times \overline{0}), 42.6,(3 \times \overline{0}), 34.9,(18 \times \overline{(3 \times \overline{0}), 38.3}), 0,0,26.1\}$ & 113 \\
(5): $\{132.69,(4 \times \overline{0}), 50.1,(4 \times \overline{0}), 46.9,(14 \times \overline{(4 \times \overline{0}), 49.3}), 0,20.9\}$ & 376 \\
(6): $\{132.69,(13 \times \overline{(5 \times \overline{0}), 54.24}), 3 \times \overline{0}, 47.53\}$ & 2125 \\
\hline
\end{tabular}

(a)

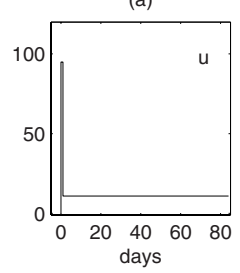

(b)

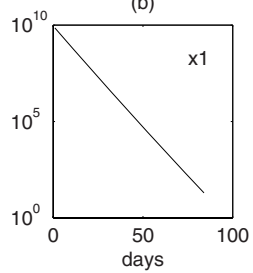

(c)

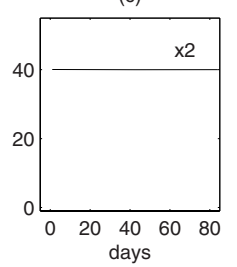

(d)

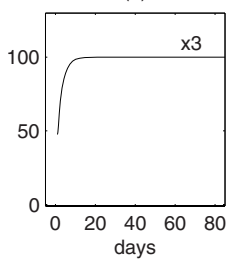

Fig. 2. The first efficient drug scheduling policy under our renewed model

In [5, we use our proposed adaptive elitist-population genetic algorithm (AEGA) to solve the drug scheduling optimization problem. Comparing with AEGA, CWGA can explore same multiple optimal solutions for the renewed model, but its efficiency (CPU time) is at least 14 times higher than that of AEGA because AEGA need to calculate the values of $x_{1}, x_{2}$ and $x_{3}$ under the differential equation system in the whole treatment period. We also combine the 
(a)

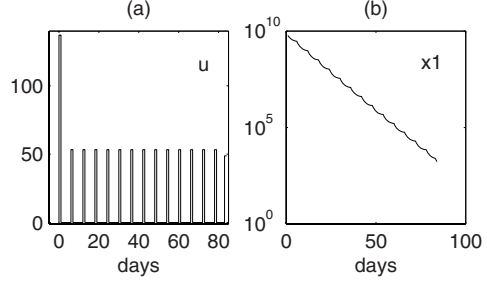

(c)

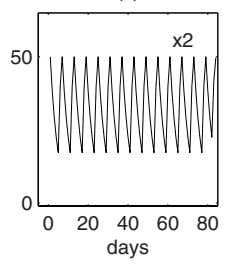

(d)

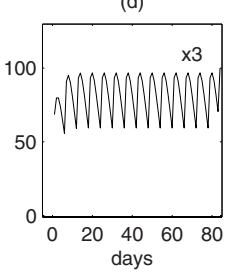

Fig. 3. The sixth efficient drug scheduling policy under our renewed model.

fitness measure of CWGA into iterative dynamic programming (IDP) to solve the renewed drug scheduling problem. IDP can find the global optimal solution and its efficiency is improved almost 9 times.

On the other hand, the multiple efficient drug scheduling policies under the new model match well with the clinical experience. In the clinical treatment, generally the drug scheduling policies include two kinds: continuous and repeated. The drug scheduling policy (1) and the drug scheduling policies (2)-(6) represent these two kinds respectively. In some patients, the aim of treatment may be to reduce the tumor size with minimum toxicity and the drug scheduling policy (6) is suitable because its cumulative drug toxicity is low and often decreases to 60 . For other patients, they may be cure despite higher toxicity, the drug scheduling policy (1) is suitable because this policy is most efficient but with high toxicity. So these multiple efficient drug scheduling policies obtained by the new model are more useful. According to the different conditions of the patients, the doctor can select the suitable drug scheduling policy to treat a cancer and get the best efficiency.

\section{Conclusion}

This paper has presented the novel fast evolutionary algorithm - cycle-wise genetic algorithm (CWGA) based on the theoretical analyses of a drug scheduling mathematical model for cancer chemotherapy. CWGA is more efficient than other existing algorithms to solve the drug scheduling optimization problem. Moreover, its simulation results match well with the clinical treatment experience, and can provide much more drug scheduling policies for a doctor to choose depending on the particular conditions of the patients. CWGA also can be widely used to solve other kinds of the real dynamic systems.

\section{Acknowledgment}

This research was partially supported by RGC Earmarked Grant 4173/04E of Hong Kong SAR and Research Grant Direct Allocation of the Shantou University. 


\section{References}

1. Carrasco, E.F., Banga, J.R.: Dynamic Optimization of Batch Reactors Using Adaptive Stochastic Algorithms. Ind. Eng. Chem. Res. 36 (1997) 2252-2261

2. Leung, K.S., Liang, Y: Genetic Algorithm with Adaptive Elitist-population Strategies for Multimodal Function Optimization. Proc. of Int. Conf. GECCO-2003. (2003) 1160-1171

3. Liang, Y., Leung, K. S., Mok, S. K., A Novel Evolutionary Drug Scheduling Model in Cancer Chemotherapy, IEEE Transactions on Information Technology in BioMedicine, 10(2), (2006) 237-245

4. Liang, Y., Leung, K. S., Mok S. K., Optimal Control of a Cancer Chemotherapy Problem with Different Toxic Elimination Processes, Proc. of IEEE WCCI. (2006) 8644-8651

5. Liang, Y., Leung, K.S., Mok S.K., Automating the Drug Scheduling with Different Toxicity Metabolism in Cancer Chemotherapy via Evolutionary Computation, Proc. of ACM GECCO-2006. (2006) 1705-1712

6. Luus, R., Harting, F., Keil, F.J.: Optimal Drug Scheduling of Cancer Chemotherapy by Direct Search Optimization. Hung. J. Ian. Chen. 23 (1995) 55-58

7. Martin, R.B.: Optimal Control Drug Scheduling of Cancer Chemotherapy. Automatica. 28 (1992) 1113-1123

8. Murray, J.M.: Optimal Control of a Cancer Chemotherapy Problem with General Growth and Loss Functions. Math. Biosci. 38 (1990) 273-287

9. Press, W.H., Teukolsky, S.A., Vetterling, W.T., Flannery, B.P.: Numerical Recipes in C: he Art of Scientific Computing. 2nd edn. Cambridge University Press (1992)

10. Tan, K.C., Khor, E.F., Cai, J., Heng, C.M., Lee, T.H.: Automating the Drug Scheduling of Cancer Chemotherapy via Evolutionary Computation. Artificial Intelligence in Medicine. 25 (2002) 169-185

11. Wheldon, T.E.: Mathematical Models in Cancer Research. Bristol. Adam Hilger. (1998) 